\title{
Self-inflicted skin burns using a deodorant spray: A
}

new case

\section{Fatima Zahra Elfatoiki, Ouafaa Bahtaoui, Hayat Skali, Fouzia Hali, Soumia Chiheb}

Department of Dermatology, Ibn Rochd UHC, 1 quartier des hopitaux 20000, Casablanca, Morocco

Corresponding author: Dr. Fatima Zahra Elfatoiki, E-mail: Fatiza59@hotmail.fr

\begin{abstract}
We report a case of self-inflicted skin burns of the left upper limb in a 11 -year-old girl with a psychological suffering, for which a deodorant spray was suspected as the probable cause the lesions. Self-inflicted burns using a cold aerosol are more common in adolescents and sustained from the spraying of aerosol onto the skin. Cooperation among dermatologists and psychiatrists is required for ensuring a good management.
\end{abstract}

Key words: Burn; Cold; Aerosol; Pathomimia; Adolescents 


\title{
Brûlures cutanées auto-infligées au spray déodorant: Un nouveau cas
}

\section{Fatima Zahra Elfatoiki, Ouafaa Bahtaoui, Hayat Skali, Fouzia Hali, Soumia Chiheb}

\author{
Department of Dermatology, Ibn Rochd UHC, 1 quartier des hopitaux 20000, Casablanca, Morocco
}

Corresponding author: Dr. Fatima Zahra Elfatoiki, E-mail: Fatiza59@hotmail.fr

\begin{abstract}
RÉSUMÉ
Nous rapportons le cas de brûlures cutanées auto-infligées du membre supérieur gauche chez une fillette de 11 ans avec une souffrance psychologique, pour lesquelles le spray déodorant était suspecté comme agent causal. Les brûlures auto-infligées par utilisation d'aérosols froids sont plus fréquente chez les adolescents et résultent de la pulvérisation d'aérosols sur la peau. La coopération entre dermatologues et psychiatres est nécessaire pour assurer une bonne prise en charge.
\end{abstract}

Mots clé: Brûlure; Froid; Aérosol; Pathomimie; Adolescents

\section{INTRODUCTION}

La pathomimie est une maladie factice provoquée consciemment par le patient lui même et dont il nie la responsabilité. Ces aspects cliniques sont multiples et variables. Nous rapportons un nouveau cas de pathomimie cutanée à type de brûlures cutanées autoinfligées par utilisation d'aérosols froids.

\section{OBSERVATION}

Patiente de 11 ans, sans antécédents pathologiques particuliers, a consulté pour des lésions cutanées du membre supérieur gauche évoluant depuis une semaine. Lexamen clinique trouvait une patiente triste avec qui le contact était difficile. Lexamen dermatologique trouvait des lésions érythémato-violacées arrondies érodées par endroit, très bien limitées, de disposition étagée le long de la face interne du membre supérieur gauche (Fig. 1). Lexamen du reste du tégument était sans particularité. Linterrogatoire minutieux avec la maman révélait des difficultés scolaires, un isolement et une agressivité chez l'enfant depuis quelques mois dans un contexte de conflit parental. Il a été noté également par la maman l'utilisation fréquente d'un spray déodorant par sa fille.
Devant ce contexte de souffrance psychologique chez la fille, la manipulation de spray déodorant, l'aspect bien dessiné des lésions cutanées et leurs localisation sur une zone accessible à la main dominante étant donné que la patiente est droitière. Nous avons pensé à une pathomimie à type de brûlures au froid par mésusage de spray déodorant. Notre diagnostic était confirmé par le pédopsychiatre chez qui la patiente était adressée pour prise en charge spécialisée. Sur le plan dermatologique un traitement symptomatique était préscrit à type de bains antiseptiques et d'applications de crèmes cicatrisante sous un pansement gras occlusif changé toutes les 48 heures par une infirmière. Une nette amélioration des lésions cutanées était notée après 2 semaines laissant place à des cicatrices achromiques (Fig. 2) .

\section{DISCUSSION}

La particularité de notre observation réside dans la rareté de la pathomimie chez l'enfant et la singularité de la méthode utilisée pour provoquer les lésions cutanées. En effet le contexte de souffrance psychologique, l'aspect clinique des lésions cutanées et leur accessibilité à la main dominante orientent vers le diagnostic.

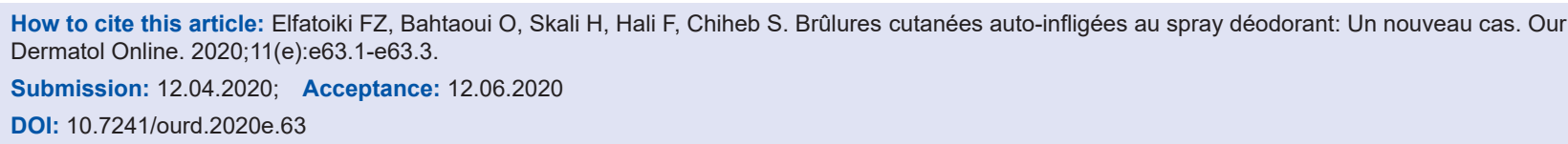




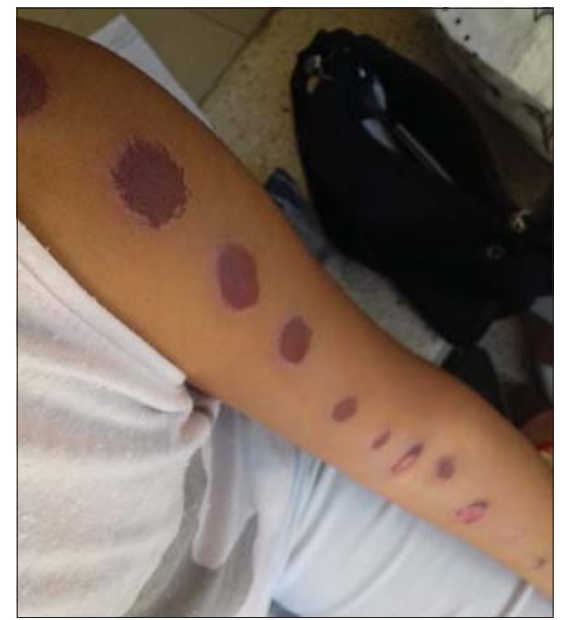

Figure 1: Lésions érythémato-violacées arrondies bien limitées du membre supérieur gauche.

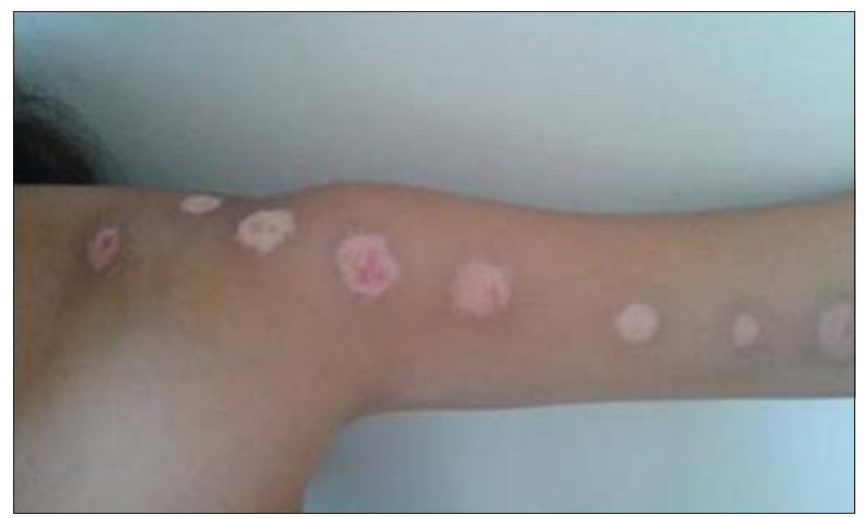

Figure 2: Cicatrices achromiques après 2 semaines.

La pathomimie ou le trouble factice correspondant à la production délibérée et consciente de symptômes d'une maladie physique ou mentale par le patient lui-même tout en niant la responsabilité, dans le but de rechercher une relation forte avec des soignants. La pathomimie cutanée étant la plus fréquente est caractérisée par des lésions uniques ou multiples, à contours bien délimités avec des aspects cliniques variable. La topographie étant très évocatrice puisque ces lésions siègent toujours à portée de la main (à gauche pour les droitiers et à droite pour les gauchers) et ne ressemblent à aucune autre lésion connue et répertoriée [1].

Les brûlures cutanées auto-infligées par utilisation d'aérosols froids sont plus fréquentes chez des adolescents avec un contexte de fragilité psychologique [2]. Ce comportement bien connu chez les adolescents sous le nom du " challenge des aérosols" résulte d'une motivation personnelle ou encouragée par les pairs sur les réseaux sociaux dans le but d'être accepté dans le groupe [3].

La brulure par utilisation d'aérosol froid se produit après pulvérisation prolongée et à courte distance de la peau. En effet, il a été démontré que la température de surface diminue à - $40^{\circ} \mathrm{C}$ après une pulvérisation d'aérosol de 20 secondes à une distance de moins de $15 \mathrm{~cm}$. Il en résulte sur la peau une brûlure par le froid qui peut être grave. Bien que ce type de brûlures n'intéresse qu'une petite surface de la peau. Sa gravité peut nécessiter des soins avancés allant jusqu'à la greffe de peau [3].

La prise en charge de ces patients se base sur la gestion de la brûlure et par la suite des cicatrices. Une prise en charge psychologique s'impose afin de lutter contre cette souffrance psychologique qui constitue le point de départ de ce comportement [4].

Nous rapportons ce nouveau cas de brûlures cutanées auto-infligées par utilisation d'aérosols froids afin de sensibiliser les cliniciens à ce type de comportement généralement plus fréquent chez les adolescents.

\section{Consent}

The examination of the patient was conducted according to the Declaration of Helsinki principles.

The authors certify that they have obtained all appropriate patient consent forms. In the form the patient(s) has/have given his/her/ their consent for his/her/their images and other clinical information to be reported in the journal. The patients understand that their names and initials will not be published and due efforts will be made to conceal their identity, but anonymity cannot be guaranteed.

\section{RÉFÉRENCES}

1. Samai-Haddadi D. La pathomimie cutanée, une perversion malmentalisée ?. Psychol Clin Projective. 2008;1:189-219.

2. Stefanutti G, Yee J, Sparnon A. Cryogenic burns from intentional use of aerosol spray in children: An emerging phenomenon. Burns. 2010;36:65-7.

3. R Maguire C, Patel B, A McBride C. Intentional self-inflicted and peer-inflicted aerosol skin injuries called 'frosties': Cohort series and systematic literature review. Aust J Gen Pract. 2018;47:477-82.

4. Ikenaga S, Nakano H, Umegaki N, Moritsugu R, Aizu T, Kuribayashi M, et al. A case of bullous dermatitis artefacta possibly induced by a deodorant spray. Dermatol. 2006;33:40-2.

Copyright by Fatima Zahra Elfatoiki, et al. This is an open access article distributed under the terms of the Creative Commons Attribution License, which permits unrestricted use, distribution, and reproduction in any medium, provided the original author and source are credited.

Source of Support: Nil, Conflict of Interest: None declared. 